\title{
Whole-system science
}

\author{
Climate change presents a tremendous opportunity for discovery that will move us towards a better \\ understanding of the physical workings of our planet and of our role as its exploiters and guardians.
}

When the Intergovernmental Panel on Climate Change released its most recent assessment report in 2007, it seemed unquestionable that its conclusion - that Earth's climate was warming rapidly and that humans were largely responsible would jolt the world into recognizing the enormity of the problem, and the need to take appropriate action. Four years on, and efforts to tackle climate change are infused with inertia, sidestepped by problems that seem more pressing or perhaps more tractable. More sobering still is the waning of public concern and political will, both of which are needed to make a sizeable dent in global greenhouse-gas emissions.

The reasons for our collective inaction are manifold and complex; a perceived lack of transparency and a loss of trust in the strength of the science caused by controversies such as the University of East Anglia's e-mail scandal have not helped to sustain support for climate policy (page 35). But neither can such controversies be held entirely accountable for our collective failure to shift from the status quo of fossil fuel consumption. Large uncertainties remain regarding the rate of change and the scale and distribution of impacts, and for the most part, we lack direct experience of those impacts - as such, we tend to see climate change as remote, not affecting us directly or any time soon (page 25). But even for those who recognize the proximity of the problem,
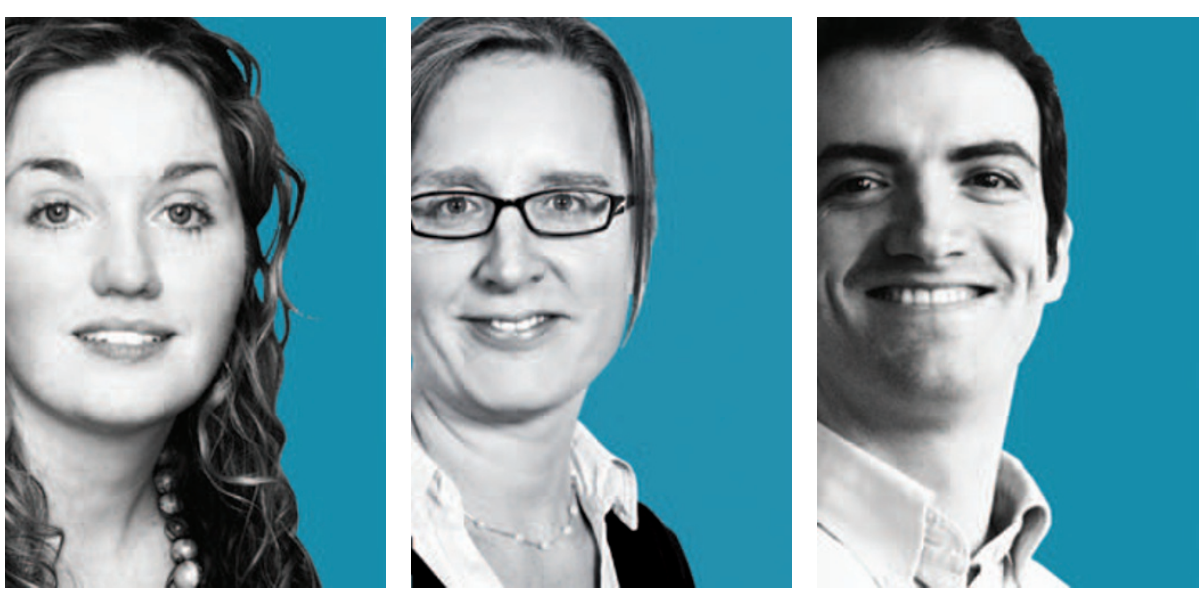

The Nature Climate Change editorial team (left to right): Olive Heffernan (Chief Editor), Jo Thorpe (Senior Editor) and Alistair Brown (Associate Editor). sustained action is difficult, especially in a regulatory vacuum. Many of us want to live low-carbon lifestyles, but research shows that we often choose convenience or comfort first, and point to others' inaction as justification for our own (page 13).

Despite the scientific consensus on climate change, much work remains to be done on understanding what the impacts of climate change will be, and how we will respond - collectively and as individuals to minimize the avoidable impacts and to adapt to those that are now inevitable. Doing so will present a challenge for society, but also a tremendous opportunity for scientific discovery - one that will ultimately move us towards a better understanding of the physical workings of our planet and our role as its exploiters and guardians. Nature Climate Change provides a forum for publishing, and discussing, the most innovative and interesting of this emerging body of research.

Like Nature and our sister journal Nature Geoscience, Nature Climate Change publishes original research on understanding change is now as much a societal problem as a physical one, Nature Climate Change will also regularly publish original social science research. The journal's first social science paper is on page 46 of this issue.

Across this broad spectrum of climate change research - from atmospheric of the physical climate system. But, as climate science to psychology - many questions at the cutting edge relate to clarifying, constraining and communicating uncertainty. Although new discoveries may narrow the uncertainty in our understanding of how the climate system and society will respond to change, they may well lead to greater uncertainty. As pointed out in the Perspective on page 35, for climate-related research to hold value for decision-making, however, policymakers and the public must understand these uncertainties. This is especially true of defining and predicting climate impacts, and so efforts to constrain, clarify and communicate those uncertainties will be a hallmark of Nature Climate Change. In this vein, our first issue features original research on the impacts of the aviation industry on the global climate (page 54), as well on the impacts of climate change on agriculture, health and economics (pages 42 and 59).

Part of the challenge of trying to advance climate research is that diverse expertise must be brought to bear to answer many of the difficult questions that are now emerging - such as how climate impacts on agriculture will affect economic growth, and how adaptation can minimize those impacts. Interdisciplinary climate research is still resource limited, both in terms of people and funding, and as with all such collaborations, it faces obstacles in agreeing a common language and a common goal. Rather than highlighting these barriers, Nature Climate Change will shine a light on success stories. In each issue, our Beyond Boundaries interview (page 68) will draw on lessons to be learnt from researchers who are taking on the specific challenge of collaborating with experts from other fields.

We hope that the presence of this new journal will draw overdue attention to the value of interdisciplinary collaborations, so that they increasingly attract more in the way of human and financial resources. Bright minds from every discipline of research will be needed to fully comprehend and tackle the problem of climate change. Nature Climate Change will encourage and communicate the very best of this work, for the benefit of science and society. 